\title{
POST-RHINOPLASTY CEREBROSPINAL FLUID LEAK CAUSED BY INTRAOPERATIVE NASAL PACKING.
}

\author{
Tiffany Munroe-Gray¹, Samit Unadkat', Catherine Rennie', Hesham Saleh1. \\ 1 Department of Otolaryngology, Charing Cross Hospital, Imperial College Healthcare NHS Trust. \\ London.
}

\section{INTRODUCTION}

Cerebrospinal fluid (CSF) leak is a very rare complication of rhinoplasty. Typically, it occurs secondary to improper handling of the perpendicular plate of the ethmoid bone ${ }^{1}$. Injuries have also been reported to the cribriform plate due to superior septal perforation during elevation of the mucoperichondrial flap using the Freer elevator ${ }^{2}$. If left untreated, it can lead to devastating intracranial sequelae ${ }^{3}$. Here, we report a previously unrecognised cause of CSF leak attributed to injudicious nasal packing.

\section{CASE}

A 19-year-old female was referred to the senior author with left-sided, clear rhinorrhea one day post cosmetic rhinoplasty surgery (done elsewhere without noted intra-operative complication). Electrophoresis was positive for beta-2 transferrin. CT imaging suggested a possible left anterior ethmoidal roof defect (Fig. 1), with mucosal thickening of ipsilateral ethmoid and maxillary sinuses (Fig. 2).

Due to persistent rhinorrhea the patient was taken to theatre the next day for Endoscopic CSF leak repair. Intraoperatively, slight damage to the axilla of the middle turbinate was noted. This was followed to where the Tilley packing forceps had caused the skull base defect. A 5x15mm defect was detected with multiple fractures of thin bone at the roof of the Ethmoid. An uncinectomy and ethmoidectomy were performed.

The repair comprised two layers of DuraGen (Integra LifeSciences, Plainsboro, New Jersey, USA) and TISSEEL glue (Baxter Healthcare Corporation, Deerfield, Illinois, USA), quadrilateral cartilage, middle turbinate mucosa from the left side, further TISSEEL glue and Gelfoam (Pfizer, New York, NY, USA). Four pieces of Nasopore (Polyganics, Groningen, The Netherlands) were used to support the repair site. This multilayer technique has had reported success of up to $93 \%$ for primary repairs ${ }^{4}$.
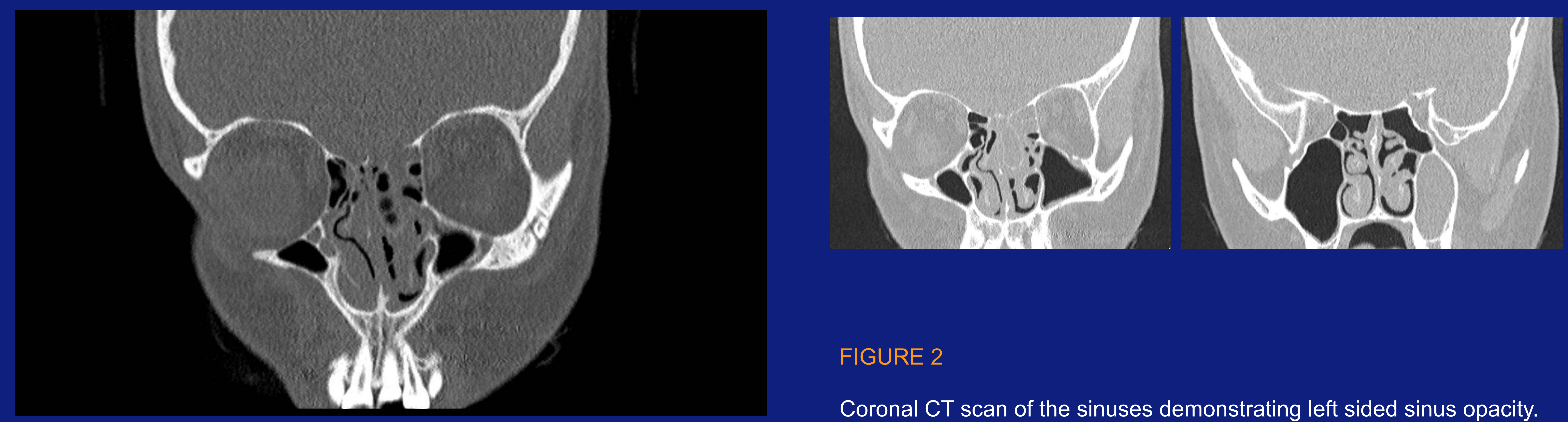

FIGURE 2

Coronal CT scan of the sinuses demonstrating left sided sinus opacity.

FIGURE 1

Coronal CT scan of the sinuses demonstrating a left sided skull base defect

\section{CONCLUSION}

\section{RESULTS}

Following 48 hours of bed rest at 30 degrees with stool softeners and straining avoidance, the patient and completed one week of co-amoxiclav. The patient remains well one year post-operatively.

This case is a pertinent reminder of the importance of nasal anatomy and an appreciation of the relationship to the skullbase is paramount when performing nasal packing. 